\title{
Caries dental y Rendimiento Académico en escolares de una Institución educativa pública peruana
}

\author{
Dental caries and academic performance in schoolchildren from a Peruvian public \\ educational institution
}

Gabriela Aracely Sanchez -Sandoval (1) ${ }^{1 a}$, Lily Diana -Zelada López ${ }^{10, b}$, Rafael Morales -Vadillo (D) ${ }^{1 \mathrm{a}, \mathrm{c}}$

\section{RESUMEN}

Objetivo: Comparar la prevalencia de caries dental mediante el índice CPOD / ceod con el rendimiento académico en escolares de 10 a 12 años de una institución educativa pública de Lima, distrito Ate. Materiales y método: Estudio observacional, analítico, trasversal, prospectivo donde participaron 136 escolares de 10 a 12 años de una escuela pública de nivel primario. Se realizó una evaluación clínica posterior a higiene dental y fue registrada en un odontograma, se utilizó el índice CPOD / ceod; el rendimiento académico se obtuvo del registro final de notas. Resultados: se encontró una alta prevalencia de caries dental en los escolares (94.9\%), predominando las lesiones moderadas (36.8\%) en comparación de dientes sanos (5.1\%). Asimismo, la prevalencia de lesiones cariosas en niños de 11 años (50.7\%), seguido de 12 años (26.5\%) y 10 años $(22.8 \%)$, se observa un incremento entre las edades de 10 años y 11 años. Además, se presentó un bajo rendimiento académico del (10.3\%) siendo la distribución en varones $(6 \%)$ y en mujeres $(3.7 \%)$. Se encontró que el ceod + CPOD son similares en niños aprobados y niños de bajo rendimiento tanto en edades como sexo, significando que no existe diferencia estadísticamente significativa entre ambas variables $(p>0.05)$. Conclusión: A pesar de la alta prevalencia de caries dental, este no presenta correlación con el bajo rendimiento académico, según el índice CPOD / ceod.

Palabras claves: Caries dental; Rendimiento académico; CPOD / ceod (Fuente: DeCS BIREME)

\section{ABSTRACT}

Objective: To compare the prevalence of dental caries through the DMFT / dmft index with the academic performance in schoolchildren aged 10 to 12 years at a public school in Lima. Methods: Observational, analytical, cross-sectional, prospective design. The sample was made up of schoolchildren aged 10 to 12 from the Fortaleza de Vitarte Educational Institution $\mathrm{N}^{\circ}$. 1239, from the period 2019. The sample size was made up of 136 schoolchildren who met the inclusion criteria. Dental evaluations were performed after dental hygiene and a record of the odontogram was taken using the DMFT / dmft index, to determine the presence of dental caries. Likewise, the record of grades was collected to obtain academic performance. Results: A high prevalence of dental caries was found in schoolchildren $(94.9 \%)$, with moderate lesions prevailing $(36.8 \%)$ compared to healthy teeth $(5.1 \%)$. Likewise, the prevalence of carious lesions in children aged 11 years $(50.7 \%)$, followed by 12 years $(26.5 \%)$ and 10 years $(22.8 \%)$, an increase is observed between the ages of 10 years and 11 years. In addition, a low academic performance of $(10.3 \%)$ was presented, being the distribution in males $(6 \%)$ and in females $(3.7 \%)$. It was found that the DMFT +dmft are homogeneous in approved children and children with low performance both in age and sex, meaning that there is no statistically significant difference between both variables $(p>0.05)$. Conclusion: Despite the high prevalence of dental caries, it does not show a correlation with low academic performance in schoolchildren aged 10 to 12 according to the DMFT / dmft index.

Key words: Dental caries; Academic performance; DMFT / dmft (Source: MeSH NLM)

Recibido: 11 de Mayo de 2021

Aprobado: 06 de Julio de 2021

En línea: 08 de Julio de 2021

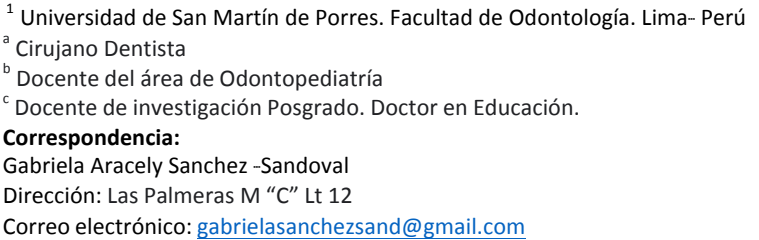

${ }^{b}$ Docente del área de Odontopediatrí

Correo electrónico: gabrielasanchezsand@gmail.com

Este es un artículo de acceso abierto distribuido bajo la licencia Creative Commons Atribución 4.0 Internacional (CC BY 4.0)

https://creativecommons.org/licenses/by/4.0/deed.es

Citar como: Sanchez Sandoval GA, Zelada López LD, Morales Vadillo R. Caries dental y rendimiento académico en escolares de nivel primario de una institución pública de Perú. KIRU. 2021 Jul-Sep; 18(3): 140- 146. https://doi.org/10.24265/kiru.2021.v18n3.02 


\section{INTRODUCCIÓN}

La caries dental es una enfermedad multifactorial que afecta a toda la población global, siendo los más afectados los niños en edad escolar y de bajos recursos económicos. Se caracteriza por ser una enfermedad dinámica, que destruye los tejidos duros del diente como resultado del desequilibrio de los ciclos de mineralización remineralización. La etiología de la caries dental fue propuesta por Paul Keyes que agrupaba; dieta, microorganismos, huésped. Asimismo, estos factores han modificado, hasta tener el modelo multinivel de Fisher-Owens, basado en los avances epidemiológicos de los últimos 25 años ${ }^{(1-9)}$.

La caries dental presenta una alta prevalencia en la población peruana, demostrado a través de estudios epidemiológicos realizados en los últimos años, siendo los niños los más afectados. Esto es debido a una higiene bucal nula o deficiente, hábitos dietéticos incorrectos, falta de acceso a servicios de salud, falta de programas sobre salud bucal etc., dando como resultado la aparición de lesiones cariosas desde edades tempranas ${ }^{(10)}$.

En el Perú, en los periodos 2001 - 2002, se encontró una prevalencia alta de caries dental $(90.43 \%)$ en escolares de nivel inicial y primaria (11). Para el 2017 los niños de 11 años (85\%) y de las edades de 3 a 4 años (76\%), presentaron una prevalencia alta de caries dental ${ }^{(12)}$. Además, Pasco, Puno y Arequipa son las regiones más afectadas por esta enfermedad ${ }^{(13)}$.

En el Perú la educación básica se divide en tres niveles, inicial, primaria y secundaria; lo cual permiten al estudiante cumplir con las competencias establecidas para cada nivel. Asimismo, el Ministerio de Educación trabaja con el Currículo Nacional de Educación Básica que permite a la plana docente lograr los objetivos trazados, buscando que los escolares adquieran y desarrollen las habilidades necesarias para su posterior inserción en la sociedad ${ }^{(14,15)}$.

El bajo rendimiento académico es un problema que atañe a la población en edad escolar. Según Cabello et al. (16) El rendimiento académico implica el cumplimiento de las metas, logros y objetivos establecidos en el programa o asignatura que cursa un estudiante, expresado a través de calificaciones que son el resultado de una evaluación que implica la superación o no de determinadas pruebas. Por ende, el rendimiento académico puede ser alterado por varios factores como: variables escolares, variables sociales y variables individuales ${ }^{(17,18)}$.

A nivel regional e internacional se realizan exámenes que miden el rendimiento académico de los escolares como: El Tercer Estudio Regional Comparativo y Explicativo (TERCE) y el Programa Internacional para la Evaluación de Estudiante (PISA). Estos exámenes nos permiten saber cómo los estudiantes están aprendiendo y los progresos que puedan tener en comparación de otras naciones ${ }^{(19,20)}$.

Un niño con lesiones cariosas puede presentar sensibilidad dentaria al momento de ingerir sus alimentos, sean fríos o calientes, dolor de los dientes, además, presenta dificultad para conciliar el sueño. Esto conlleva que el niño llegue a la escuela cansado y no pueda participar activamente en las actividades que se presenten en el salón de clases o en el patio de juegos con sus demás compañeros. El ausentismo escolar se puede presentar si la lesión cariosa se encuentra en estadios avanzados; debido a la presencia de dolor u otras molestias, conllevando que el escolar pierda clases, afectando su rendimiento académico ${ }^{(13)}$.

El objetivo de este estudio fue comparar la prevalencia de caries dental mediante el índice CPOD / ceod con el rendimiento académico en escolares de 10 a 12 años de una institución educativa pública de Lima.

\section{MATERIALES Y MÉTODOS}

El estudio fue de tipo observacional, analítico, trasversal, prospectivo donde la muestra fue conformada por 136 escolares de 10 a 12 años de $5^{\text {to }}$ y $6^{\text {to }}$ de primaria. Se realizó en la Institución Educativa $N^{\circ} 1239$ Fortaleza de Vitarte en el distrito de Ate, Lima. El tipo de muestreo fue no probabilístico por conveniencia, los criterios de inclusión fueron: Estudiantes de 10 a 12 años, niños cuyos padres firmaron el consentimiento informado y escolares que firmaron el asentimiento. Asimismo, los criterios de exclusión fueron: Escolares que no asistieron a la evaluación, con habilidades diferentes, enfermedades sistémicas y estudiantes repitentes. 
El estudio se dividió en dos partes: la primera parte consistió en una evaluación clínica de las lesiones de caries dental en los niños. La evaluación clínica se realizó en el salón de clases

designándose un espacio el cual fue acondicionado, además, de contar con protocolos de bioseguridad. Los escolares recibieron cepillo y pasta dental para realizar el cepillado; el mismo que fue supervisado y corregido por el examinador. Se contó con luz natural y artificial, el escolar fue ubicado en una colchoneta que se colocó encima de una mesa para una mejor atención. Para la evaluación de la cavidad bucal se utilizó equipo de examen básico ${ }^{(21)}$. Se procedió a secar las piezas dentarias con gasa, permitiendo una mejor visualización de las lesiones de caries, los datos fueron registrados en una ficha. El diagnóstico de caries dental se realizó con el índice CPOD y ceod ${ }^{(20)}$. Para el análisis del estudio, se tomó como resultado la suma de los valores ceod + CPOD por individuo, clasificándolo de la siguiente forma: Grave (CPOD + ceod > 7), Moderado (CPOD + ceod $=4-6)$, Leve $($ CPOD + ceod $=1-3)$, Sano $($ CPOD + ceod $=0)$.

La segunda parte consistió en recolectar las notas finales de los estudiantes; las cuales fueron obtenidas del Sistema de Información de Apoyo a la Gestión Educativa (SIAGIE) ${ }^{22}$, y fueron entregadas por el director en forma física al terminar el año escolar. Para el análisis del rendimiento académico, se trabajó con la Situación final que es el resultado de las competencias alcanzadas por el escolar durante un año electivo, asimismo, se clasificó en:
Aprobado y Bajo rendimiento; siendo este último el que agrupa a los escolares que requieren recuperación pedagógica y desaprobados.

Los datos obtenidos fueron trasladados a una base de datos en el programa de EXCEL; posteriormente en el programa estadístico de SPSS versión 25, se empleó estadística descriptiva para hallar los porcentajes, las frecuencias absolutas y relativas, medias y desviación estándar; y pruebas de correlación mediante la prueba de $U$ de Mann-Whitney tanto para edad como para sexo.

\section{RESULTADOS}

Fueron evaluados 136 escolares, de los cuales 64 fueron niños y 72 fueron niñas; de acuerdo al grupo etáreo se hallaron 31 niños de 10 años, 15 de sexo masculino y 16 de sexo femenino; 69 niños de 11 años, 32 de sexo masculino y 37 de sexo femenino; y, 36 niños de 12 años, 17 de sexo masculino y 19 de sexo femenino.

Se encontró una alta prevalencia de caries dental (94.9\%), donde las lesiones moderadas fueron las más comúnmente encontradas con (36.8\%), seguida de lesiones graves (32.4\%) y leves (25.7\%); asimismo, solo (5.1\%) de los niños evaluados no presentaban lesiones cariosas.

La población escolar evaluada presentó CPOD mas ceod alto en los escolares de 11 años (50.7\%), seguido de 12 años (26.5\%) y 10 años (22.8\%). Asimismo, se observa que hay un incremento de lesiones de caries en los escolares de 11 años en relación a los escolares de 10 años. (Tabla 1 ).

Tabla 1. Prevalencia de caries dental correspondiente al índice CPOD + ceod en escolares de 10 a 12 años según edad en la Institución Educativa №1239 Fortaleza de Vitarte - 2019

\begin{tabular}{ccccccccc} 
CPOD + ceod & \multicolumn{2}{c}{10 años } & \multicolumn{2}{c}{11 años } & \multicolumn{2}{c}{12 años } & \multicolumn{2}{c}{ TOTAL } \\
\cline { 2 - 9 } & $\mathrm{n}$ & $\%$ & $\mathrm{n}$ & $\%$ & $\mathrm{n}$ & $\%$ & $\mathrm{n}$ & $\%$ \\
\hline $\begin{array}{c}\text { Sano } \\
\text { CPOD + ceod }=0\end{array}$ & 1 & $0.7 \%$ & 4 & $2.9 \%$ & 2 & $1.5 \%$ & 7 & $5.1 \%$ \\
\hline $\begin{array}{c}\text { Leve } \\
\text { POD + ceod = 1-3 }\end{array}$ & 11 & $8.1 \%$ & 17 & $12.5 \%$ & 7 & $5.1 \%$ & 35 & $25.7 \%$ \\
\hline $\begin{array}{c}\text { Moderado } \\
\text { POD +eod = 4-6 }\end{array}$ & 10 & $7.4 \%$ & 26 & $19.1 \%$ & 14 & $10.3 \%$ & 50 & $36.8 \%$ \\
\hline $\begin{array}{c}\text { Grave } \\
\text { CPOD + ceod }>7\end{array}$ & 9 & $6.6 \%$ & 22 & $16.2 \%$ & 13 & $9.6 \%$ & 44 & $32.4 \%$ \\
\hline Total & 31 & $22.8 \%$ & 69 & $50.7 \%$ & 36 & $26.5 \%$ & 136 & $100 \%$
\end{tabular}


En cuanto al rendimiento académico, se observa que el total de escolares aprobados es de $(89.7 \%)$, mientras que los escolares con bajo rendimiento académico son (10.3\%). Asimismo, en el grupo de escolares de 10 años se encontró 29 niños aprobados (93.5\%), 2 niños con rendimiento bajo (6.5\%); en los escolares de 11 años, 60 niños aprobados (87\%), 9 niños con bajo rendimiento académico (13\%); para finalizar, en los escolares de 12 años, 33 niños aprobados (91.7\%), 3 niños con bajo rendimiento (8.3\%).
(Tabla 2). No se encontraron diferencias estadísticamente significativas al relacionar la presencia de caries dental de acuerdo a la edad; teniendo un valor $p$ de $0.113,0.141$ y $p=0.729$ para los 10, 11 y 12 años de edad, respectivamente. (Gráfico 1). No se encontraron diferencias estadísticamente significativas al relacionar la presencia de caries dental de acuerdo al sexo; teniendo un valor $\mathrm{p}$ de de 0.168 y 0.276 para sexo masculino y sexo femenino respectivamente. (Gráfico 2)

Tabla 2. Prevalencia del rendimiento académico en escolares de 10 a 12 años según edad de la Institución Educativa N ${ }^{\circ} 1239$ Fortaleza de Vitarte - 2019

\begin{tabular}{ccccccccc} 
Rendimiento & \multicolumn{2}{c}{10 años } & \multicolumn{2}{c}{11 años } & \multicolumn{2}{c}{12 años } & \multicolumn{2}{c}{ TOTAL } \\
\cline { 2 - 9 } académico & $\mathrm{n}$ & $\%$ & $\mathrm{n}$ & $\%$ & $\mathrm{n}$ & $\%$ & $\mathrm{n}$ & $\%$ \\
\hline Aprobado & 29 & $93.5 \%$ & 60 & $87 \%$ & 33 & $91.7 \%$ & 122 & $89.7 \%$ \\
Bajo rendimiento & 2 & $6.5 \%$ & 9 & $13 \%$ & 3 & $8.3 \%$ & 14 & $10.3 \%$ \\
\hline Total & 31 & $100 \%$ & 69 & $100 \%$ & 36 & $100 \%$ & 136 & $100 \%$
\end{tabular}
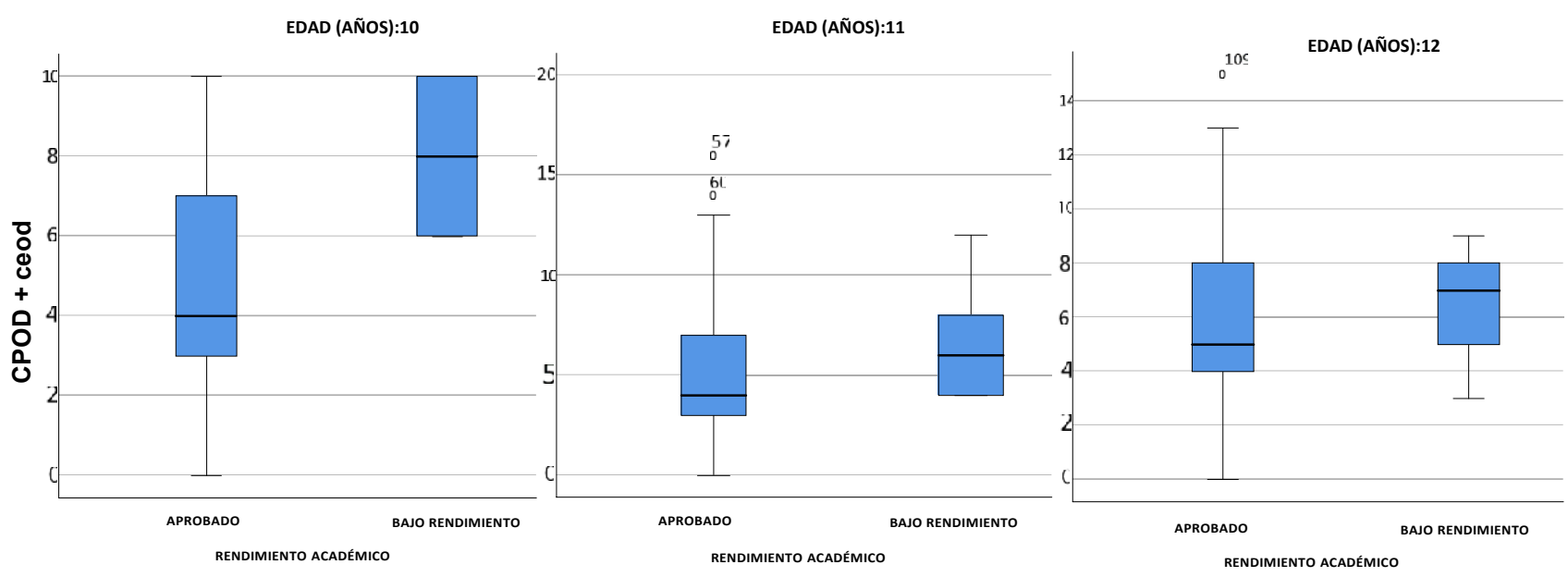

RENDIMIENTO ACADÉMICO

RENDIMIENTO ACADÉMICO

RENDIMIENTO ACADÉMICO

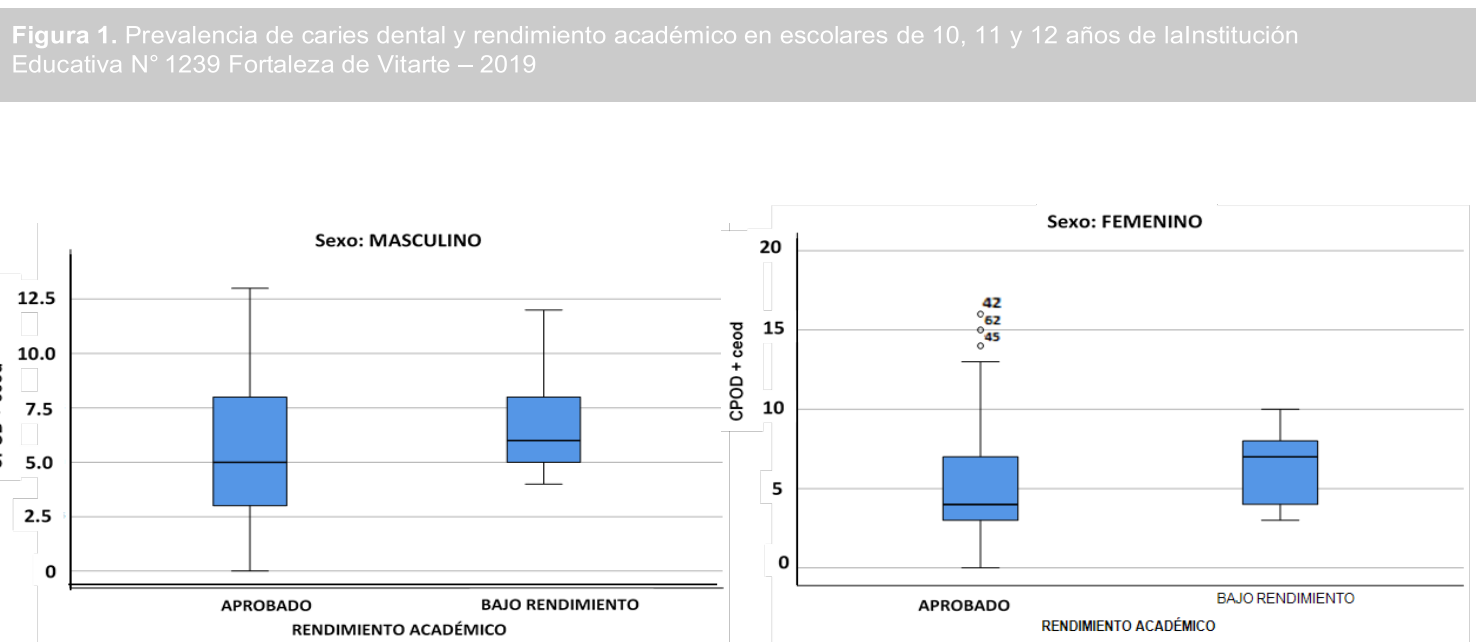




\section{DISCUSIÓN}

La caries dental es una enfermedad multifactorial, que se manifiesta en las estructuras dentales como resultado del desequilibrio de los ciclos de desmineralización - remineralización ${ }^{(1,2)}$. Su alta prevalencia en escolares es causada por una higiene bucal deficiente, hábitos dietéticos incorrectos como alta ingesta de alimentos azucarados, entre otros ${ }^{(5,6)}$. En algunos casos, la presencia de caries dental puede influenciar en el bajo rendimiento académico de los niños; de acuerdo a esto, la presente investigación se basa en comparar la prevalencia de caries dental y rendimiento académico en escolares.

En relación a los resultados obtenidos en la distribución muestral, se encontró un porcentaje mayor de mujeres $(52.8 \%)$ con respecto a los varones $(47.7 \%)$; lo opuesto a lo encontrado por Matamare $M$, donde se evidencia un mayor porcentaje de varones con $(55.1 \%)$ y mujeres (44. 9\%) ${ }^{(23)}$. Asimismo, Vallejo $R$ encontró una proporcionalidad parecida a la presente investigación donde el grupo predominante son las mujeres (60.4\%), seguida de los varones $(39.6 \%)^{(24)}$.

Con respecto a la prevalencia de lesiones de caries, se observó que el $94.9 \%$ de los escolares presenta caries dental; se encontró mayor prevalencia de lesiones moderadas de caries con un $36.8 \%$, seguida lesiones graves con $32,4 \%$ y lesiones cariosas leves con $25.7 \%$; es importante resaltar que existe un porcentaje mínimo de escolares sanos con $5.1 \%$, esto nos demuestra que las medidas de control de la caries dental aún no logran obtener un beneficio en la mayoría de la población, especialmente en los círculos sociales de mayor riesgo, como es la población escolar que participó de este estudio. Así mismo, se puede observar un incremento del CPOD más ceod en escolares de 11 años $(50.7 \%)$, en relación a la edad de 10 años $(22.8 \%)$, esto significa un incremento exponencial de lesiones de caries entre ambas edades. Los resultados hallados coinciden con el levantamiento muestral realizado en el país por el Ministerio de Salud (MINSA) donde se encontró que el $85 \%$ de los niños de 11 años presentaban caries dental ${ }^{(11)}$.
Se observan valores similares en los índices CPOD y ceod, en los estudios de Maharani et al, donde se encontró que los niños de 10 a 12 años presentan un índice alto de caries dental $(90 \%)$; y Matamare $M$, quien separa a los escolares por grados, de 5to y 6to de primaria los que coinciden con las edades de 11 a 12 años, su índice de CPOD es alto $(60.4 \%)^{(24,25)}$.

Con respecto al rendimiento académico se encontró un gran número de escolares aprobados $(89.7 \%)$, y pocos escolares con rendimiento bajo (10.3\%). Asimismo, las calificaciones aprobatorias se presentan mayormente en niños de 11 años 60 casos, seguidos de 12 años 33 casos y 10 años 29 casos. De acuerdo al sexo las calificaciones aprobatorias se presentan mayormente en mujeres $(49.3 \%)$ en comparación con los varones (44.4\%); es en este grupo donde se presenta mayor prevalencia de rendimiento académico de tipo bajo con un $(6 \%)$, en comparación al de las mujeres que presentan (3.7\%). Al comparar nuestros resultados con los trabajos de Vallejo $R$ y Matamare $M$; se observa diferencias en la variable analizada, debido a que la recolección de notas fue de forma diferente a la nuestra ${ }^{(23,24)}$. Dichos autores dividen en cuatro los valores de rendimiento académico: DAR (Dominan los Aprendizajes Requeridos), AAR (Alcanzan los Aprendizajes Requeridos), PAAR (Parcialmente Alcanza los Aprendizajes Requeridos), NAAR (No Alcanza los Aprendizajes Requeridos) y trabajan con las notas de primero y segundo semestre, mientras las nuestras son anuales, conllevando resultados diferentes ${ }^{(23,24)}$.

Al asociar la prevalencia de caries dental con el rendimiento académico en escolares; no se observó diferencias estadísticamente significativas en los grupos etáreos de 10, 11 y 12 años; teniendo un valor $p$ de $0.113,0.141 \mathrm{y}$ 0.0729 , respectivamente. Dichos resultados son incompatibles con el trabajo de Matamare donde se puede observar una asociación entre un CPOD muy bajo (29.5\%) y rendimiento académico satisfactorio, CPOD bajo (10.3\%) y rendimiento académico en progreso y CPOD moderado $(10.3 \%)$ y rendimiento en progreso. Matamare concluye que la mitad de los escolares presenta un índice CPOD y ceod alto con un rendimiento académico no satisfactorio. Asimismo, Maharani et al., Paula y Vallejo R, manifiestan que presentan una relación 
significativa entre estas dos variables, con valores $p<0.005^{(23-25)}$. Con referencia a la asociación entre la prevalencia de caries dental y rendimiento académico según sexo, no se encontraron diferencias estadísticamente significativas en hombres $(p=0.276)$ ni en mujeres $(p=0.168)$. En el trabajo de Vallejo $R$, se encontró un resultado similar en el grupo de varones, donde los resultados no presentan diferencia estadística significativa $(p=0.296)^{(24)}$.

Las variaciones que puede presentar un trabajo de investigación se deben a múltiples factores como tamaño de la muestra, formas diferentes al momento de recolectar los datos, escales de mediciones, múltiples variables, lo que conlleva a tener resultados diferentes en relación al presente trabajo de investigación ${ }^{(26,27)}$. En el caso del presente estudio, el número reducido de niños que presentaba un bajo rendimiento académico nos ha limitado la posibilidad de encontrar una diferencia significativa al momento de analizar las variables.

Se concluye que la prevalencia de caries dental en los escolares evaluados fue alta: $94.4 \%$, solo contando con $5.1 \%$ de sanos. Además, las lesiones moderadas fueron las más persistentes seguidas de lesiones graves. Finalmente, al comparar la prevalencia de caries dental con el rendimiento académico, se concluyó que no existe diferencia estadísticamente significativa entre las dos variables, donde ceod / CPOD es similar en aprobados y en bajo rendimiento tanto para edades como sexo.

Asimismo, mencionar que este artículo, es el resultado de la investigación de una tesis para optar el título de cirujano dentista, la cual se encuentra publicada en el repositorio de esta casa de estudios.

Contribución de autoría: GASS y LDZL diseñaron el estudio; GASS participó en la recolección de datos. GASS, LDZL y RMV analizaron los datos. Los autores redactaron el manuscrito, realizaron la revisión crítica y aprobaron la versión final.

Fuentes de financiamiento: autofinanciado.

Conflicto de Interés: Los autores no declararon conflictos de interés.

\section{REFERENCIAS BIBLIOGRÁFICAS}

1. Duque C, Caldo-Teixeira A, Ribeiro A, Ammari M,Abrau F,Antunes L. Odontopediatria: Uma visão contemporânea.São Paulo:Livraria Santos Editora; 2013.

2. Pitts NB, Zero DT, Marsh PD, Ekstrand K, Weintraub JA, Ramos-Gomez F, etal. Dental caries. Nat Ver Dis Primers. 2017; 3:17 030. 3:17030. doi: 10.1038/nrdp.2017.30.

3. Bordoni N,Escobar A,Castillo R. Odontología Pediátrica. La salud bucal del niño y el adolecente en el mundo actual. Buenos Aires: Médica panamericana; 2010.

4. Henostroza G, Arana A, Bernabé E, Bussadori S, Calderón V, Delgado L, Et al. Caries dental principios y procedimientos para el diagnóstico. Lima: Universidad Cayetano Heredia; 2007.p 172.

5. Uribe Echevarría J. Operatoria dental. Madrid. Ediciones Avances Médico-dentales;1990.

6. Fejerskov O. Concepts of dental caries and their concequences for understanding the disease. Community Dent Oral Epidemiol.1997;25(1):5-12.

7. Keyes $\mathrm{PH}$. The infectious and transmisible nature of experimental dental caies. Findings and implications. Arch Oral Biol.1960; 1:304320.

8. Newbrun E. Cariology. $2^{\mathrm{a}}$ edición, Baltimor: Lippincott Williams and Wilkins;1983.

9. Fisher-Owens AS, Gansky SA, Platt LJ, Weintraub JA, Soobader MJ, Bramlett MD, Newacheck W. Influences on children's oral health: a conceptual model. Pediatrics.2007; 120(3):510-20.DOI: 10.1542/peds.2006-3084.

10. Gopalan T, Asokan S, John JB, Geetha Priva PR. School absenteeism, academic performance and self-esteem as proxy measures of oral health status: A crosssectional study. J Indian Pedod Prev Dent. 2018; 36(4)339-346

11. Gob.pe. Plataforma única del estado. Más de 342 mil escolares recibirán atención en salud bucal. Lima;2015.

12. Gob.pe. Plataforma única del estado. Minsa: $85 \%$ de niños de 11 años tiene caries dental por inadecuada higiene bucal. Lima;2017.

13. Gob.pe. Plataforma única del estado. Pascó, Puno y Arequipa son las regiones con mayor prevalencia de caries dental en niños de 3 a 15 años. Lima;2017.

14. Ministerio de Educación. Currículo universal, de la educación básica. Lima; Dirección de imprenta; 2017. 3 -51 p.

15. Klein H,Palmer CE. Studies on dental caries. VII. Sex Differences in dental caries experience of elementary schoolchildren. Public Health Rep. 1938; 53: 1685-90. 
16. Cabello C, Abello R, Palacio J. Relación de Burnout y el rendimiento académico con la satisfacción frente a los estudios en estudiantes universitarios. Av. Psicol Clin Latino. 2007; 25(2): 98-111.

17. Ministerio de Educación. Currículo universal, de la educación básica. Lima. Dirección de imprenta. 2017; 3 -51.

18. Dellucia K. Dinámica familiar y rendimiento escolar. Buenos Aires: Bibloss; 2014.

19. Flotts MP, Manzi J, Jimenez D, Abarzúa A, Cayuman C, Garcia MJ. Informe del resultado TERCE: Logros de aprendizaje. Rev UNESCO.2016; p 3-141.

20. División de Educación, BID. La Realidad del Desarrollo Social Latinoamericano: Avances y Desafíos de la educación en América Latina y el Caribe. Washington D.C: Banco Interamericano de Desarrollo;2017. p 2- 96.

21. Organização Mundial da Saúde. Levantamentos em saúde bucal: métodos básicos. 5a ed. São Paulo: FOUSP. 2017; p.1:32-33.

22. Ministerio de Educación. Aprobación de nóminas de matrícula mediante el siagie;2017.

23. Hatamare M. Relación de la enfermedad caries en el rendimiento académico en niños de 06-12 años de edad en la institución educativa 40135
"Teniente Ferre". [Tesis para optar el título de cirujano Dentista] Arequipa: Universidad Católica de Santa María;2017.

24. Vallejo Aguirre R. Calidad de vida en relación a la salud bucal y rendimiento académico en escolares de 11 años de edad del Cantón Azores-Ecuador. [Tesis para optar el grado de maestría]. Lima: Universidad Cayetano Heredia;2016.

25. Maharani D, Adiatman M, Rahardjo A, Burnside $G$, Pine C. An assessment of the impacts of child oral health in Indonesia and associations with self-esteem,school performance and perceived employability. BCM oralHealth.2017; (21): 17-65.

26. Maisanche Lema D. Estudio de la relación entre salud oral y el rendimiento académico en escolares de la unidad educativa Galileo Galilei, perteneciente al Cantón Rumiñahui. [Tesis para optar el grado de Magister]. Ecuador: Universidad Central de Ecuador; 2016.

27. Paula J, Lisboa C,Meneghim M, Pereira A, Ambrosama G, Mialhe F. School performance and oral health conditions : analysis of the impact mediated by socio-economic factors. Int JPaldiatr Dent. 2016; (26):52-9.

Gabriela Aracely Sanchez Sandoval

ORCID (1) https://orcid.org/0000-0002-1427-4278

gabrielasanchezsand@gmail.com

Lily Diana Zelada López

ORCID (1) http://orcid.org/0000--0002--7636--7971

Izeladal @usmp.pe

Rafael Morales Vadillo

ORCID (1) https://orcid.org/0000-0002--7835-6408

rmoralesv@usmp.pe 